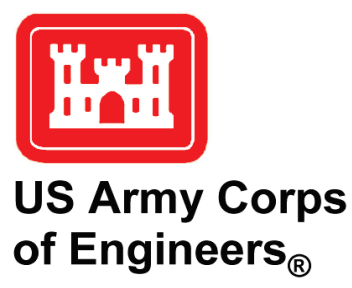

\title{
Sediment Scaling for Mud Mountain Fish Barrier Structure
}

\author{
by Jeremy A. Sharp, Gary L. Brown, and Gary L. Bell
}

PURPOSE: This Coastal and Hydraulics Laboratory technical note describes the process of scaling sediment for the 1:20 General Physical Model of the Fish Barrier Structure downstream of Mud Mountain Dam, which is located on the White River near Seattle, WA.

INTRODUCTION: The White River is a mountainous river with head waters on Mount Rainier. The Fish Barrier Structure on the White River serves as a collection point of migratory fish. Operators at the structure collect the fish and transport them upstream of Mud Mountain Dam. Currently, the fish barrier uses a weir to create a head differential, which stops the passage of the migrating fish. However, the head difference causes additional deposition upstream of the fish barrier. Furthermore, with large annual sediment loads, the White River is naturally aggrading, and the fixed weir does not allow for structure operations to mobilize the sediment. Thus, a new structure is desired to both mitigate sediment accumulation and increase fish collection capacity.

Correctly selecting the appropriate physical model sediment is critical for properly simulating the prototype behavior of the proposed structure (Franco 1978). Numerous studies (Carr et al. 2015; Einstein and Chien 1956; Henriquex et al. 2008; Kocyigit et al. 2005; Lu et al. 2013; Yalin 1989) have expended extensive effort in an attempt to replicate prototype sediment behavior in movable bed models. Properly capturing the movable bed freedom is imperative when channel modifications or complex flow patterns alter bed alignment. This freedom is directly dependent on appropriately scaled model sediment.

For this model, there are two basic sediment behaviors that are of principal interest:

- the stability of the coarse sediment bed (i.e., probability of erosion/scour)

- the deposition of fine sediment from suspension (wash load).

To represent these two behaviors in a single physical model, it was determined that the best approach was to select two separate sediment classes to be modeled: one bed material class that is scaled for initiation of motion and one suspended sediment class that is scaled for deposition from suspension.

EFFECTIVE GRAIN SIZE: The inherent complexities associated with sediment gradations cannot (in general) be properly scaled in a physical model. Therefore, a simplification is introduced whereby an effective grain size is estimated for the prototype mixture. The effective grain size is the size required for a single grain-sized model sediment to behave the same as a prototype sediment gradation, with respect to some desired characteristic (Christensen 1969). For the purposes of this model, the desired characteristic is the probability of motion. Once an effective grain size is defined based on the prototype gradation, this effective grain size is scaled for model application. 
The sediment scaling analysis for this effort was guided by the need to determine which prototype sediment classes would be modeled as bedload and which would be modeled as suspended load. The prototype sediment is generally subdivided into two groups: wash load (WL) and bed material load (BML). These two components comprise the total sediment load. The WL is the sediment in transport derived from sources other than the bed (Lane 1947). The WL is finer than the BML and is not found in appreciable quantities in the bed (Biedenharn et al. 2006). Conversely, the BML is sediment whose particles are found in appreciable quantities in the bed (Vanoni 2006; Biedenharn et al. 2006). However, the delineation of what percentage constitutes an appreciable quantity is abstract and challenging (Biedenharn et al. 2006). Because the definitions of WL and BML are qualitative, additional interpretation to discretely define these components is required.

For this effort, the delineation between WL and BML is defined by the criteria of Einstein (1950) as the D10 (grain size in which $10 \%$ of the particles are smaller) of the mixture, in which WL and BML are finer and coarser than $10 \%$ of the sediments by gradation, respectively. This analysis should be based on a representative prototype gradation taken from non-slack water areas (Einstein 1950). For this study, the sediment sampling was limited to locations downstream of the existing fish barrier structure. Figure 1 presents the sieve analysis for two downstream samples (500 feet downstream of barrier) of the prototype sediment. Taking the (numerical) derivative of the percent passing curve of the representative prototype gradation results in the fraction of each class in the sample by sieve size. The fractional analysis reveals a clear bimodal distribution of the prototype sediment (Figure 2). This bimodal distribution suggests that simulating the sediment behavior of the prototype with two separate sediment classes is appropriate.

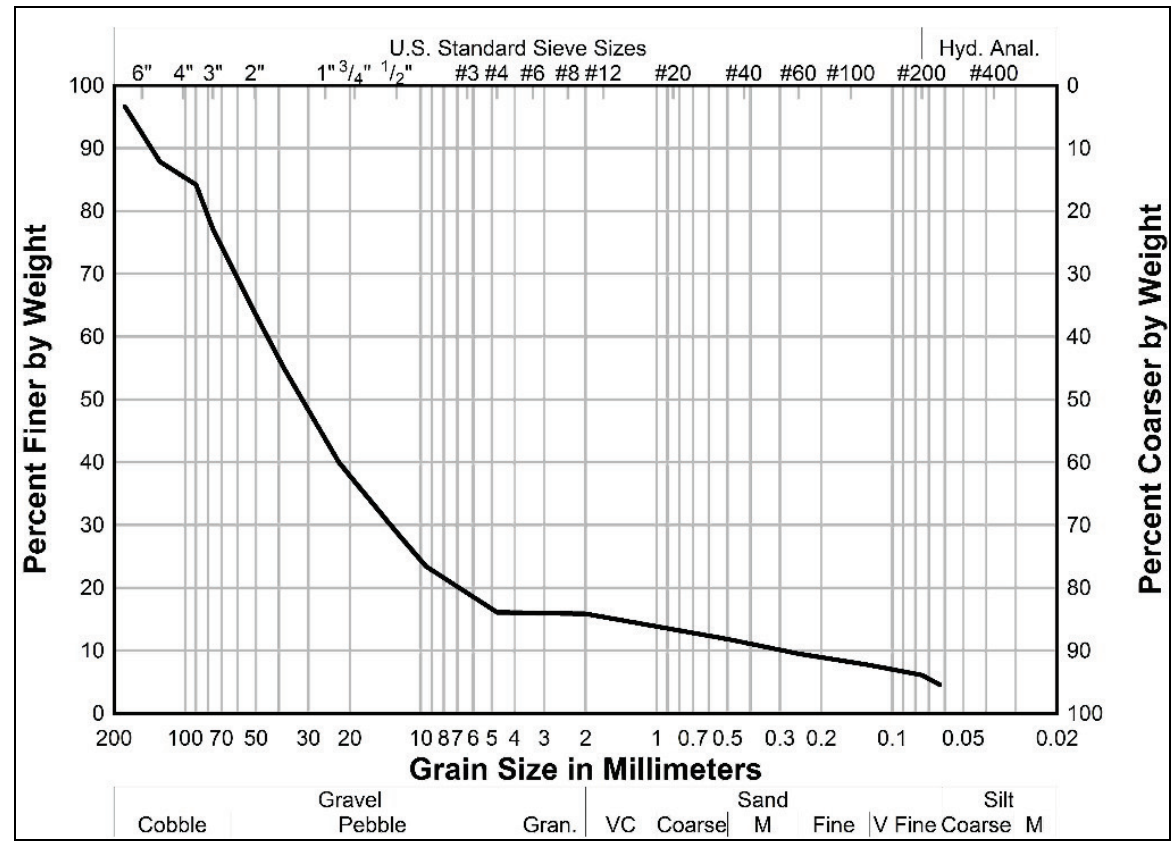

Figure 1. Representative sediment sample (eight samples) collected from downstream of the existing Fish Blockage Structure, White River WA. 


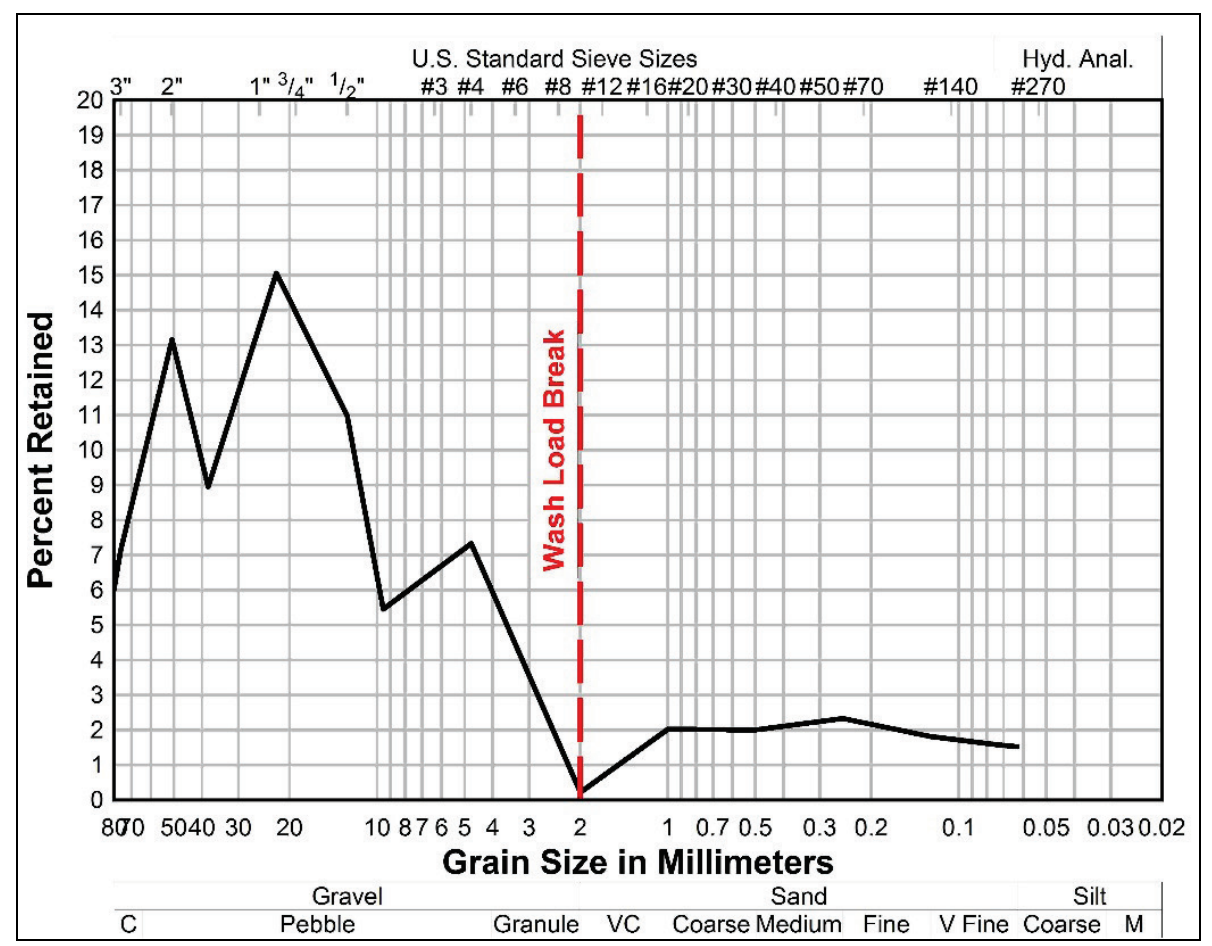

Figure 2. Percent retained from representative sediment sample.

The bimodal break was 2 millimeters (mm), which corresponds to the D10 of one of the samples (Figure 1). Here, the coarser material ( $>$ D10) is designated as the BML while the finer material $(<\mathrm{D} 10)$ is designated as the WL. By separating the two quantities into their own respective gradations and using Equation 1 (Christensen 1969), the effective grain size $\left(\mathrm{d}_{\mathrm{e}}\right)$ is calculated for each representative class. The effective grain sizes for the White River downstream of the Mud Mountain Fish Barrier Structure are found from eight samples to be $29.5 \mathrm{~mm}$ (coarse gravel) and $0.27 \mathrm{~mm}$ (medium sand) for BML and WL, respectively (see nomenclature at end of text for variable definitions).

$$
\frac{1}{d_{e}}=\int_{0}^{1} \frac{d_{y}}{d_{s}}=\sum_{n=1}^{n=p} \frac{y_{n}-y_{n-1}}{\ln \frac{d_{s n}}{d_{s n-1}}} \times\left[\frac{1}{d_{s n-1}}-\frac{1}{d_{s n}}\right]
$$

SIMILITUDE: Free surface flow similitude can be defined as follows:

We say that two flows are similar if they are geometrically similar and if all the relevant dimensionless parameters are the same for both flows. (Stockstill 2015)

Similitude principles form the design fundamentals such that the results of the model can be converted to prototype. For any model, free surface similitude requires the existence of geometric, kinematic, and dynamic similitude. Geometric similitude exists between the model and prototype if the ratios of all corresponding dimensions in the model and prototype are equal. Kinematic similitude exists between model and prototype if the flow paths of the moving particles are geometrically similar and if the ratios of the particle velocities are equal. Dynamic 
similitude exists within geometrically and kinematically similar systems when the ratio of all homologous forces in the model and prototype are equal.

Unfortunately, it is not possible to achieve true dynamic similitude for all forces in a scaled physical model (ASCE 2000). However, most physical systems are dominated by a small number of the forces. If two dominant forces can be identified, the non-dimensional ratio of these forces can be scaled, ensuring dynamic similitude of these forces. ${ }^{1}$

The non-dimensional term that defines dynamic similitude for free-surface flow is the Froude number, where the ratio of inertia to gravity is equal between the model and prototype. Particle similitude can be evaluated in terms of two governing force ratios:

- incipient motion (Shields similitude)

- suspension and settling (Rouse similitude) (ASCE 2000).

Julien (2002) outlined four important parameters for moveable bed similitude:

- Froude similitude

- resistance/roughness similitude; bed roughness similitude is critical for similar hydrodynamics from prototype to model such as water slope and velocity

- similitude of dimensionless grain diameter, defining sediment grain regime

- similitude of the bed entrainment of individual grains into suspension.

By correctly accounting for these four parameters defined by Julien (2002), it is possible to acquire physical model results applicable to the prototype. However, perfect similitude for sediment and flow is unattainable in a physical model, so the dominant forces must be identified and properly scaled (i.e., enforced). For this study, Shields similitude will be enforced for the BML (to ensure that the initiation of motion is scaled properly), and Rouse similitude will be enforced for the WL (to ensure that suspension and deposition are scaled properly).

The selection of model sediment classes that satisfy these criteria requires the use of four dimensionless numbers:

- Rouse number (or particle Peclet number) (Equation 2)

- Shields parameter (Equation 3)

- dimensionless grain diameter (Equation 4)

- particle Reynolds number (Equation 5).

The equations describing these terms are given below. The following sections will discuss how they are used to select the model sediments.

$$
P=\frac{\omega_{s}}{\kappa U_{*}}
$$

\footnotetext{
${ }^{1}$ Note that, for any physical model, the neglected forces are still important as they determine the physical limitations on scale. For example, Froude models must be large enough to remain turbulent (Reynolds constraint) and to ensure that surface tension does not become significant in the model (Weber constraint).
} 


$$
\begin{gathered}
\tau_{*_{c}}=\frac{\tau_{c}}{\left(\gamma_{s}-\gamma\right) d} \\
d_{*}=d_{m}\left[\frac{g(S G-1)}{v^{2}}\right]^{1 / 3} \\
\mathbb{R}_{e^{*}}=\frac{U_{*} d}{v}
\end{gathered}
$$

BED MATERIAL LOAD SCALING: By applying the effective grain size, the BML for the prototype was scaled for initiation of motion. The defining non-dimensional parameter for initiation of motion is the Shields parameter (Shields 1936) (Figure 3). It is common practice in physical modeling to assume that the Shields parameter is a constant between model and prototype: that is, the critical shear stress in the model and the prototype scales only with the grain diameter and with the specific gravity of the sediment. For this study, the prototype sediment consists of gravel and cobble. Geometrically scaling the prototype effective grain size results in a sand size gradation. A sand and gravel-based sediment does not in general have the same Shields parameter. Therefore, it is necessary to ascertain the appropriate Shields parameter for the prototype sediments.



Figure 3. Shields diagram (Shields 1936).

The most direct way to obtain the Shields parameter is to determine directly the critical shear stress of both the prototype sediments (BML and WL) by performing flume tests and then calculate the Shields parameter from Equation 3. However, for this study, flume testing of the prototype sediment mixture was not a viable option since the D90 of the prototype is nearly 180 
$\mathrm{mm}$ and would require an estimated unit discharge 35 cubic feet/second/foot to mobilize. This discharge is beyond the discharge capacity of the available test facilities.

Since direct measuring of the critical shear stress of the prototype sediment proved impractical, a novel, alternative method for estimating the Shields parameter was developed for this study. It can be shown from first principles that the Shields parameter is a function of the angle of repose of the sediment (Christensen 1993). The angle of repose is defined as the maximum or critical angle (Bagnold 1941) when a granular mass destabilizes and slides (Carrigy 1970; Deganutti et al. 2011). Several methods for determining the Shields parameter indicate the dependency of the Shields parameter on the angle of repose (e.g., Julien [1995]). Therefore, for a given sediment mixture, the Shields parameter can be estimated indirectly by measuring the angle of repose for the mixture.

To measure the angle of repose of the prototype sediment, a tilt-tank apparatus was designed and fabricated. The tilt-tank testing apparatus is an acrylic box with a removable and sealable top. The sediment sample is loosely placed in the box and submerged in water. Once the sample is saturated, the box is tilted at a rate of 0.2 degrees (deg)/second. Particle sifting can occur, but this is not considered failure. A failure is said to occur when multiple individual particles are dislodged at the same time. Table 1 shows the synopsis of the results for a series of 11 tests of the prototype sediment conducted for this study.

\begin{tabular}{|c|c|c|c|c||c||}
\hline \hline Table 1. Tilt-tank test results for the prototype bed material load. \\
\hline \hline \multicolumn{5}{|c|}{ Prototype Bed Material Load Analysis } \\
\hline \hline $\begin{array}{c}\text { Bed Layer } \\
\text { Thickness, } \mathbf{m m}\end{array}$ & $\begin{array}{c}\text { Average Angle of } \\
\text { Repose, deg }\end{array}$ & $\begin{array}{c}\text { Standard } \\
\text { Deviation, deg }\end{array}$ & \multicolumn{3}{|c|}{ Gradation, $\mathbf{~ m m}$} \\
\hline \hline 165.1 & 46.4 & 2.49 & 0.7 & 27.9 & 125.6 \\
\hline 241.3 & 45.3 & 3.55 & 0.6 & 27.9 & 113.4 \\
\hline
\end{tabular}

The values for the angle of repose are larger than those reported by Deganutti (2011). For a mixture of $22.4<\mathrm{d}_{50}<31.5 \mathrm{~mm}$, the average angle of repose was $40.8 \mathrm{deg}$ (Deganutti 2011). However, the gradation was more uniform and was described as rounded. Carrigy (1970) showed that the angle of repose for a uniformed submerged natural material was less than the same material in air (also noted by Land [1964]). These gradations were not as widely distributed as the present prototype material.

Once the angle of repose was defined for the prototype material, the model material was scaled. First, the Shields number for the prototype material was calculated using Equation 3. Likewise, it was calculated for the model. However, an initial guess at the effective grain size of the model was required. This was done by geometrically scaling the prototype effective grain size by $\lambda(1: 20)$. Once both the model and prototype Shields number were estimated, Equation 6 was applied:

$$
d_{m}=\lambda \frac{\tau_{c^{*} p}}{\tau_{c^{*} m}} d_{p}
$$

This provided a new estimate for $d_{m}$ (model sediment diameter) and was substituted into Equation 4. The dimensionless grain size was calculated and used in Equation 7 Julien (2010): 


$$
\tau_{*_{c m}}=0.06 \tan \phi_{m} \text { When } d_{*}>50
$$

Then the new estimate for the models Shields number was re-applied in Equation 6 to repeat the iteration. The iteration was looped until $d_{m}$ stopped changing.

In order to characterize the sensitivity of the above calculation to uncertainties in the input parameters, a perturbation analysis was performed. The variation in particle sizing that resulted from this perturbation analysis is shown in Table 2. Perturbations of both the effective grain size and angle of repose of the prototype sediments were performed. The effective grain size was varied by $+/-20 \%$ and $+/-40 \%$. Justification for the wide range in variation in effective grain size arises from the observed tendency for skewing of large gradation sizes toward the smaller end. This skew is due primarily to the large granular material and the difficulty in obtaining a representative sample. Variation in the angle of repose was based on the mean (45.3 deg) and $+/$ one standard deviation found from the tilt-tank testing.

\begin{tabular}{|c|c|c|}
\hline \multirow[b]{2}{*}{ Phi } & \multicolumn{2}{|c|}{ Grain Size $\left(d_{e}\right), m m$} \\
\hline & Prototype & Model \\
\hline 441.8 & 29.5 & 2.19 \\
\hline 45.3 & 29.5 & 2.48 \\
\hline 48.9 & 29.5 & 2.81 \\
\hline 41.8 & 23.6 & 1.84 \\
\hline 45.3 & 23.6 & 1.98 \\
\hline 48.9 & 23.6 & 2.25 \\
\hline 41.8 & 35.4 & 2.63 \\
\hline 45.3 & 35.4 & 2.97 \\
\hline 48.9 & 35.4 & 3.37 \\
\hline 41.8 & 41.3 & 3.07 \\
\hline 45.3 & 41.3 & 3.47 \\
\hline 48.9 & 41.3 & 3.94 \\
\hline
\end{tabular}

From the above analysis, a very fine gravel material was selected as the best option for the 1:20 model. The selected material has a D50 of $3.2 \mathrm{~mm}, \mathrm{D} 10$ of $2.5 \mathrm{~mm}$, and a D90 of $4.6 \mathrm{~mm}$. Thus, the lambda (1), or scale ratio, for the models BML is 0.11 . This $I$ is much greater than the $I$ selected for the model, but this is not of concern since the Shields criterion only requires that the critical shear stress is scaled. The effective roughness height for prototype is based on the D90 (van Rijn 1984). The average D 90 for the prototype is $100 \mathrm{~mm}$, so the 1 for model roughness is 0.046 . This is slightly less than the geometric 1 of 0.05 (1:20 scale) but is arguably sufficient due to the physical limitations of the model.

WASH LOAD SCALING: The WL was scaled for Rouse similitude. The assumption is that there is enough energy in the system (model) to keep the WL suspended until it encounters an area of momentum loss (e.g., behind the barrier). Satisfaction of Rouse similitude requires that the settling velocity is scaled such that Froude similitude is satisfied. This will also ensure that the model has consistent scaling with the vertical velocity and the shear velocity. Froude 
similitude requires that the velocity scales as the square root of the length scale (Equation [8]). Hence, Rouse similitude requires that the settling velocity of the model WL sediment scales as the square root of the settling velocity of the prototype WL sediment.

$$
w_{s . m .}=\frac{1}{\sqrt{ } 20} w_{s . p .}=0.2236 w_{s . p .}
$$

The effective grain size of the WL in the prototype is $0.26 \mathrm{~mm}$ (settling velocity is $32.7 \mathrm{~mm} / \mathrm{s}$, Julien [2002]). Scaling the fall velocity of the effective size results in a model WL grain size of $0.1 \mathrm{~mm}$. However, a grain size of $0.1 \mathrm{~mm}$ is a fine sediment and is challenging to procure. The closest available material has a D50 of $0.17 \mathrm{~mm}, \mathrm{D} 10$ of $0.11 \mathrm{~mm}$, and a D90 of $0.28 \mathrm{~mm}$. The settling velocity of this material is $17.7 \mathrm{~mm} / \mathrm{s}$ compared to the required $7.3 \mathrm{~mm} / \mathrm{s}$ (Julien 2002). Alternative materials (specific gravity $<2.65$ ) were also evaluated but would be cost prohibited due to the volume of material required for model testing.

CONCLUSION: The nature of the sediment in the prototype creates an advantage for sediment scaling. The bimodal behavior of the prototype sediment allows for the use of two different sediment sizes in the model, thus allowing for scaling one representative size for Shields (initiation of motion) and one for Rouse (settling). Congruently, the large prototype material allows for sediment scaling without model or material distortions. This allows for the realistic three-dimensional prototype behavior in the model, yielding a properly scaled analysis of scour and sedimentation around the modeled structure.

Validation of the model sediment behavior was limited to qualitative observation from site experts (both Seattle District hydraulic engineers and the fish barrier operators). At a flow of approximately 4,000 cubic feet/second, the bed mobilizes and readily moves. A similar mobilization occurred in the model for the BML model sediment. While not definitive, the similar BML mobilization point, between prototype and model, provides some confidence in the scaled sediments performance. Also, there has been no validation for the WL model sediment.

Application of the method should be limited to physical modeling of granular non-cohesive sediments where a bimodal prototype gradation exists. Alternatively, if there is no bimodal gradation but a dominance or interest in studying either initiation of motion or settling, then the method for the desired behavior can be used independently.

\section{NOMENCLATURE}

$$
\begin{aligned}
& d=\text { grain size } \\
& d_{e}=\text { effective grain size } \\
& d_{s}=\text { grain size } \\
& d_{*}=\text { dimensionless grain size } \\
& g=\text { acceleration of gravity } \\
& \gamma=\text { specific weight of water } \\
& \gamma_{s}=\text { specific weight of sediment }
\end{aligned}
$$


$\lambda=$ geometric scale ratio

$\mathbb{R}_{e^{*}}=$ particle Reynolds number

$S G=$ specific gravity

$\tau_{c}=$ critical shear stress

$\theta_{c}$ or $\tau_{* c}=$ Shields number

$U_{*}=$ friction velocity

$v=$ kinematic viscosity

$P=$ Rouse number

$\phi=$ Angle of Repose

$y=$ percentage passing for $d_{s}$

$w_{s}=$ settling velocity

ADDITIONAL INFORMATION: This technical note was prepared by Jeremy A. Sharp, research hydraulic engineer at the U.S. Army Engineer Research and Development Center, Coastal and Hydraulics Laboratory. Questions about this technical note can be addressed to Mr. Sharp at 601-634-4212 or Jeremy.A.Sharp@usace.army.mil.

This CHETN should be cited as follows:

Sharp, J. A., G. L. Brown, and G. L. Bell. 2017. Sediment scaling for Mud Mountain Fish barrier structure. ERDC/CHL CHETN-VII-16. Vicksburg, MS: U.S. Army Engineer Research and Development Center. http://dx.doi.org/

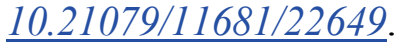

\section{REFERENCES}

American Society of Civil Engineers (ASCE). 2000. Hydraulic modeling concepts and practice. ASCE Manuals and Reports on Engineering Practice No. 97. Reston, VA: ASCE.

Bagnold, R. A. 1941. The physics of blown sand and desert dunes. London: Methuen.

Biedenharn, D. S., L. C. Hubbard, C. R. Thorne, and C. C. Watson. 2006. Understanding sediment sources, pathways and sinks in regional sediment management: Wash load and bed-material load concept. ERDC-TNSWWRP-06-3. Vicksburg, MS: U.S. Army Engineer Research and Development Center.

Carr, K. J., A. Ercan, and M. L. Kavvas. 2015. Scaling and self-similarity of one-dimensional unsteady suspended sediment transport with emphasis on unscaled sediment material properties. Journal of Hydraulic Engineering 141.

Carrigy, M. 1970. Experiments on the angles of granular materials. Sedimentology 14(3-4):147-158.

Christensen, B. A. 1969. Effective grain size in sediment transport. Proc. 12th Congr. Int. Assoc. Hydraul. Res. $3: 223-231$.

Christensen, B. A. 1993. Class notes for "Sediment Transport." University of Florida.

Deganutti, A., P. Tecca, and R. Genevois. 2011. Characterization of friction angles for stability and deposition of granular material. Italian Journal of Engineering and Environment: 5th International Conference on DebrisFlow Hazards: Mitigation, Mechanics, Prediction and Assessment, Padua, Italy, 313-318. 
Einstein, H. A. 1950. The bed-load function for sediment transportation in open channel flows. Tech. Bull. 1026. Washington, DC: U.S. Dept. Agriculture.

Einstein, H., and N. Chien. 1956. Similarity of distorted river models with movable beds. Trans. Am. Soc. Civ. Eng. 121(1):440-462.

Franco, J. J. 1978. Guidelines for the design, adjustment and operation of models for the study of river sedimentation problems. Instruction Report H-78-1, U.A. Vicksburg, MS: U.S. Army Corps of Engineers, Waterways Experiment Station.

Henriquez, M., A. Reniers, B. Ruessink, M. Stive, T. Stanton, and D. Foster. 2008. On the scaling of sediment transport in the nearshore. In Proc., $2^{\text {nd }}$ Int. Conf. on the Application of Physical Modeling to Port and Coastal Protection - Coastlab '08, International Association for Hydro-Environmental Engineering and Research, Bari, Italy, 193-204.

Julien, P. Y. 2010. Erosion and sedimentation. Cambridge: Cambridge University Press.

Julien, P. Y. 2002 River mechanics. Cambridge: Cambridge University Press.

Kocyigit, O., B. Lin, and R. A. Falconer. 2005. Modeling sediment transport using a lightweight bed material. Marit. Eng. 158(1):3-14.

Land, L. S. 1964. Eolian cross-bedding in the beach dune environment, Sapelo Island, Georgia. J. Sediment. Petrol. 34(2):389-394.

Lane, E. W. 1947. American Geophysical Union. Subcommittee on sediment terminology report. American Geophysical Union Transactions 28(6):936-38, December.

Lu, J., X. Liao, and G. Zhao. 2013. Experiment study of effect of geometric distortion upon suspended sediments in bending channels. Sediment. Geol. 294(10):27-36.

Shields, A. F. 1936. Application of similarity principles and turbulence research to bed-load movement. Vol 26, 524. Berlin, Germany: Mitteilungen der Preussischen Versuchsanstalt f€ur Wasserbau und Schiffbau.

Vanoni, V. A. 2006, Sedimentation engineering: Manuals and reports on engineering practice No. 54. Reston, VA: American Society of Civil Engineers Publications.

Van Rijn, L. C. 1984. Sediment transport, part I: bed load transport. Journal of hydraulic engineering 110(10):1,431-1,456.

Yalin, M. 1989. Fundamentals of hydraulic physical modelling. Recent Advances in Hydraulic Physical Modelling. NATO ASI series E: Applied sciences, ed. R. Martin, Vol. 165, 1-37. Dordrecht, Netherlands: Kluwer Publishers.

NOTE: The contents of this technical note are not to be used for advertising, publication,

or promotional purposes. Citation of trade names does not constitute an official

endorsement or approval of the use of such products. 